Published in final edited form as:

Clin Rheumatol. 2016 April ; 35(4): 1071-1076. doi:10.1007/s10067-015-3152-2.

\title{
Overweight Older Adults, Particularly After an Injury, are at High Risk for Accelerated Knee Osteoarthritis: Data from the Osteoarthritis Initiative
}

\author{
Jeffrey B. Driban ${ }^{1}$, Charles B. Eaton ${ }^{2}$, Grace H. Lo ${ }^{3,4}$, Lori Lyn Price ${ }^{5,6}$, Bing Lu ${ }^{7}$, Mary F. \\ Barbe $^{8}$, and Timothy E. McAlindon ${ }^{1}$ \\ ${ }^{1}$ Division of Rheumatology, Tufts Medical Center, 800 Washington Street, Box \#406, Boston, MA \\ 02111, USA \\ ${ }^{2}$ Center for Primary Care and Prevention, Alpert Medical School of Brown University, 111 \\ Brewster Street, Pawtucket, RI 02860, USA \\ ${ }^{3}$ Medical Care Line and Research Care Line, Houston Health Services Research and \\ Development (HSR\&D) Center of Excellence Michael E. DeBakey VAMC, Houston, TX, USA \\ ${ }^{4}$ Section of Immunology, Allergy, and Rheumatology, Baylor College of Medicine, 1 Baylor Plaza, \\ BCM-285, Houston, TX 77030, USA \\ ${ }^{5}$ The Institute for Clinical Research and Health Policy Studies, Tufts Medical Center, Boston, MA \\ 02111, USA \\ ${ }^{6}$ Tufts Clinical and Translational Science Institute, Tufts University, 800 Washington Street, Box \\ \#63, Boston, MA 02111, USA \\ ${ }^{7}$ Brigham \& Women's Hospital and Harvard Medical School, 75 Francis Street PBB-B3, Boston, \\ MA 02115, USA \\ ${ }^{8}$ Department of Anatomy and Cell Biology, Temple University School of Medicine, 3500 North \\ Broad Street, Philadelphia, PA, 19140
}

\section{Abstract}

Objectives-We explored whether age and body mass index (BMI) can help identify a subset of individuals who are at high risk for accelerated knee osteoarthritis (AKOA) compared with common knee osteoarthritis (KOA).

\begin{abstract}
Method-In the Osteoarthritis Initiative, a multicenter observational cohort study of KOA $(\mathrm{n}=4,796)$, we studied participants without KOA at baseline (Kellgren-Lawrence $[\mathrm{KL}]<2)$. Participants could have 1 of 3 outcomes: 1) AKOA: $\geq 1$ knee progressed to end-stage KOA within 48 months, 2) common KOA: $\geq 1$ knee increased in radiographic scoring within 48 months
\end{abstract}

\footnotetext{
"Corresponding Author: Jeffrey B. Driban, PhD, ATC, CSCS, Division of Rheumatology, Tufts Medical Center, 800 Washington Street, Box 406, Boston, MA 02111. jeffrey.driban@tufts.edu, Phone: 617-636-7449, Fax: 617-636-1542.

Conflicts of Interest: The authors declare they have no conflicts of interest with regard to this work.

ETHICAL STANDARDS

Institutional review boards at each OAI clinical site and the OAI coordinating center (University of California, San Francisco) approved the OAI study and all participants provided informed consent prior to participating in the OAI.
} 
(excluding those with AKOA), and 3) no KOA: no change in KL grade in either knee. After verifying an interaction between age, BMI, and recent knee injury, we determined if we could identify a specific subset of individuals at high risk for AKOA instead of KOA. First, we reviewed 3-dimensional graphs with age, BMI, and probability of AKOA versus KOA on the axes. We then conducted a logistic regression with AKOA as the outcome and age-BMI groups as the predictor.

Results-In our main analyses we found that older individuals with a BMI $<35 \mathrm{~kg} / \mathrm{m}^{2}$ were more likely to develop AKOA than common KOA ( $\mathrm{n}=64$; mean [SD] BMI $=27.3[3.1] \mathrm{kg} / \mathrm{m}^{2}$; odds ratio $=3.47,95 \%$ confidence interval $=1.70$ to 7.10 ), especially if they had a recent knee injury.

Conclusions-While older age and greater BMI are independently associated with AKOA, we found that older individuals who had a higher BMI, particularly if they have an injury, were more likely to develop AKOA than common KOA.

\section{Key Indexing Terms}

body mass index; osteoarthritis; age; injuries; knee

\section{INTRODUCTION}

Knee osteoarthritis (KOA) is typically a slowly progressive disorder; however, a subset of knees progress with dramatic rapidity (e.g. from normal appearance to end-stage disease within 4 years). Three to four percent of individuals in the Osteoarthritis Initiative (OAI) without radiographic KOA at baseline develop accelerated KOA (AKOA) in the first 4 years of the study [1]. Among knees with AKOA approximately 65\% progress in less than 12 months from no radiographic KOA (Kellgren-Lawrence $[\mathrm{KL}]$ grade $=0$ or 1 ) to advanced KOA (KL 3 or 4), which was defined by the development of a definite osteophyte and joint space narrowing. Individuals with AKOA report greater knee symptoms and functional disability than individuals with common KOA [2]. Greater age, higher body mass index (BMI), and new knee injuries were key risk factors for AKOA [1]. However, age, BMI, and knee injury are also risk factors for KOA in general. To identify who is at risk for AKOA, it is vital to determine if a specific subset of individuals is more likely to develop AKOA instead of the more common KOA that develops gradually.

Therefore, we explored age and BMI to identify a subset of individuals who are at higher risk for AKOA compared with common KOA. As a preliminary first step, we tested whether there was an interaction between age, BMI, and knee injury. These findings may help us identify a subset of individuals at most risk for AKOA and lead to more rigorous monitoring of these populations.

\section{MATERIALS AND METHODS}

We used data from the OAI, which is a multicenter observational cohort study of KOA that collected longitudinal clinical and image data [3] annually from 4,796 participants over a four-year follow-up period [4]. Institutional review boards at each OAI clinical site and the coordinating center approved the OAI study and all participants provided informed consent. 


\section{Participant Selection}

Among participants without baseline radiographic KOA (KL Grade<2) in either knee we evaluated three groups: 1) AKOA: at least one knee progressed to advanced-stage KOA (KL Grade 3 or 4) within 48 months, 2) common KOA: at least one knee increased in radiographic scoring within 48 months (excluding those defined as AKOA), and 3) no KOA: no change in KL grade in either knee between baseline and 48-month follow-up. More details on this study sample are published elsewhere [1].

\section{Knee Radiographs}

Study staff obtained weight-bearing, bilateral, fixed-flexion, posterior-anterior knee radiographs at baseline and the annual OAI visits. Central readers, who were blinded to sequence of follow-up radiographs, scored paired images for KL grades. The agreement for these readings (read-reread) was good (weighted kappa $=0.70$ to 0.78 ). These data are publicly available (Files: kXR_SQ_BU\#\#_SAS; version 0.6, 1.6, 3.5, 5.5, and 6.3))[4].

\section{Clinical Data}

Demographic, anthropometric, and other participant characteristics, which we selected $a$ priori as potential risk factors or covariates, were acquired based on a standard protocol [4]. We extracted several key baseline variables: age, BMI, sex, blood pressure, and knee alignment [1]. Baseline knee alignment was based on a goniometric measurement while the participant stood [4]. Normal alignment was defined as 0 degrees and any deviation was defined as varus or valgus malalignment.

\section{Self-Reported Knee Injury}

At each annual visit participants were asked "Since your last annual visit to the OAI clinic about 12 months ago, have you injured your right knee badly enough to limit your ability to walk for at least two days?". A similar question was asked for the left knee. Among individuals with accelerated or common KOA we focused on injuries to the knee that progressed. Among individuals with no KOA we evaluated if the participant had a knee injury to either knee. Based on prior work [1], we focused on injuries within one year of the study outcome. We defined the study outcome visit for each group as follows: 1) AKOA: the first visit with a KL grade of 3 or 4,2) common KOA: the first visit with an increase in KL grade, and 3) no KOA: the 48-month OAI visit (see Figure 1).

\section{Statistical Analyses}

Before exploring cut-offs for age and BMI, we first tested whether there was an interaction between age, BMI, and recent knee injury. We used a multinomial logistic regression to determine if the outcome of accelerated or common KOA was associated with 8 groups, which we defined based on a combination of age, body mass index, and injury: 1) normalweight (BMI $<25 \mathrm{~kg} / \mathrm{m}^{2}$ ), younger (45-64 years) without recent knee injury; 2) normalweight, younger, with recent knee injury; 3 ) normal-weight, older ( $\ 65$ years), without recent knee injury; 4) normal-weight, older, with recent knee injury; 5) overweight (BMI $\geq 25 \mathrm{~kg} / \mathrm{m}^{2}$ ), younger, without recent knee injury; 6) overweight, younger, with recent knee injury; 7) overweight, older, without recent knee injury; and 8) overweight, older, with 
recent injury. The reference group was younger participants with normal weight and without a recent knee injury. The reference outcome was no KOA. The regression was adjusted for sex, baseline presence of static knee malalignment, and systolic blood pressure. We selected these variables because they differed at baseline among the individuals with AKOA, common KOA, versus no KOA [1].

We reviewed 3-dimensional surface and contour graphs with age (continuous), BMI (continuous), and probability of AKOA versus common KOA on the axes to identify groups that have the highest probability of AKOA compared with common KOA (see Figure 2a). We also explored surface and contour graphs with age and BMI categorized by standardized cut-offs (age in 5-year intervals, BMI in $2.5-\mathrm{kg} / \mathrm{m}^{2}$ intervals, Figure $2 \mathrm{~b}$ ). These graphs were reviewed in all individuals who develop AKOA or common KOA and in those with or without a knee injury. We omitted the group with no KOA because we thought it was relevant to identify a subset of individuals that was more likely to develop AKOA than more gradual KOA progression. After we identified two possible subsets (red boxes in Figure 2b) as high risk we conducted a logistic regression with AKOA as the outcome and age-BMI groups as the predictor (adjusting for sex and recent knee injury). We also explored stratified analyses among those with and without a recent knee injury. All analyses and plots were completed in SAS 9.3 (Cary, NC, USA).

\section{RESULTS}

We previously described the baseline characteristics of OAI participants with no KOA $(\mathrm{n}=1325)$, common KOA $(\mathrm{n}=187)$, and AKOA $(\mathrm{n}=54)$ [1]. Overall, 58\% were female and $74 \%$ had static knee malalignment (varus or valgus). Table 1 provides the distribution of individuals by age-BMI-injury group among those with accelerated, common, or no KOA. The interaction of BMI, age, and knee injury by the outcome of OA status was highly significant $(\mathrm{p}<0.0001)$. Four groups were more likely to experience AKOA instead of no KOA compared with normal-weight, younger individuals without a recent knee injury: 1) normal-weight, older, with recent knee injury (odds ratio $[\mathrm{OR}]=76.0 ; \mathrm{n}=8$ ); 2) overweight, older, with a recent knee injury $(\mathrm{OR}=25.9 ; \mathrm{n}=15) ; 3)$ overweight, younger, with a recent knee injury ( $\mathrm{OR}=22.8 ; \mathrm{n}=39)$; and 4$)$ overweight, older, without a recent knee injury $(\mathrm{OR}=4.5 ; \mathrm{n}=299$; see Table 1$)$.

\section{Primary Results}

After verifying that there was an interaction between age, BMI, and recent knee injury, we tested if there was a subset of individuals that was more likely to develop AKOA than common KOA (see Figures $2 \mathrm{a}$ and $2 \mathrm{~b}$ ). We identified two high-risk sets of individuals: 1 ) individuals $>65$ years of age with BMI $<35 \mathrm{~kg} / \mathrm{m}^{2}(\mathrm{n}=64,27 \%$, mean [SD] age $=70.5$ [3.3] years, mean $B M I=27.3[3.1] \mathrm{kg} / \mathrm{m}^{2}$ ) and 2) individuals $<65$ years of age with $\mathrm{BMI}>32.5$ $\mathrm{kg} / \mathrm{m}^{2}\left(\mathrm{n}=43,18 \%\right.$, mean age $=55.1[4.6]$ years, mean $\mathrm{BMI}=34.7[2.4] \mathrm{kg} / \mathrm{m}^{2}$; red boxes in Figure $2 b$ ). Among the 64 individuals $>65$ years of age with BMI $<35 \mathrm{~kg} / \mathrm{m}^{2}$, only $17(27 \%)$ were considered normal weight (BMI $<25 \mathrm{~kg} / \mathrm{m}^{2}$ ) in our initial analyses. We defined all other participants as low risk. Overall, older individuals with a BMI $<35 \mathrm{~kg} / \mathrm{m}^{2}$ were almost 3.5 times more likely to develop AKOA instead of common KOA than individuals at low 
risk (Table 2). In contrast, the 43 younger individuals with a BMI $>32.5 \mathrm{~kg} / \mathrm{m}^{2}$ were not statistically more likely to develop AKOA instead of common KOA (Table 2). These findings were consistent among those with and without a recent knee injury; however, we found the largest odds ratios among older individuals with a BMI $<35 \mathrm{~kg} / \mathrm{m}^{2}$ and an injury $(n=9 ;$ Table 2).

\section{DISCUSSION}

We found that older individuals with a BMI $<35 \mathrm{~kg} / \mathrm{m}^{2}$, especially if they had a recent knee injury, were more likely to develop AKOA than common KOA. In contrast, younger individuals with $\mathrm{BMI}>32.5 \mathrm{~kg} / \mathrm{m}^{2}$ were not more likely to develop AKOA than common KOA.

While it may seem paradoxical that older individuals with a BMI $<35 \mathrm{~kg} / \mathrm{m}^{2}$ had the highest odds of developing AKOA instead of common KOA it is noteworthy that younger individuals with a high BMI had a large incidence of both common KOA and AKOA. Hence, in this subset we could not determine who would develop AKOA instead of more gradual KOA progression.

It is also important to consider the two other risk factors among older individuals with a BMI $<35 \mathrm{~kg} / \mathrm{m}^{2}$. First, 7 (29\%) of the 24 older individuals with a lower BMI who develop AKOA had a knee injury, compared with only 2 (5\%) out of 40 that developed common KOA (Table 2). Secondly, $73 \%$ of older individuals with a BMI $<35 \mathrm{~kg} / \mathrm{m}^{2}$ were overweight $\left(\geq 25 \mathrm{~kg} / \mathrm{m}^{2}\right.$ ). Therefore, this high-risk group had a high prevalence of other risk factors: recent knee injuries and being overweight.

Knee injuries disrupt biomechanics and structural integrity of a knee and represent a key risk factor of KOA and AKOA [1]. Knee injuries may be particularly devastating to joint health among older and/or overweight individuals. Our findings support evidence that anterior cruciate ligament transection in older rats lead to an accelerated onset of cartilage damage compared with younger rats [5]. Furthermore, a high-fat diet, which is associated with being overweight, may lead to a quicker onset of cartilage damage, synovitis and possibly osteophytes among mice with induced meniscal pathology [6,7]. The faster onset of pathologic changes were independent of body weight $[6,7]$. This may indicate that other factors influence the accelerated changes; such as, elevated inflammation [8-13] or glucose homeostasis [10,14-16]. Future studies should further explore mediators that may explain how age and high BMI influence the onset of AKOA.

We acknowledge that these are exploratory analyses that have two key limitations: limited sample size and use of self-reported injury data. In the main analyses, only 64 individuals were older with a BMI $<35 \mathrm{~kg} / \mathrm{m}^{2}$ and 34 adults were younger with a BMI $232.5 \mathrm{~kg} / \mathrm{m}^{2}$. The sample sizes were even smaller among groups in our preliminary analyses and secondary analyses, where we accounted for the status of recent knee injury. We pursued these analyses, despite the limited sample size, because we believe it was extremely important to understand if there may be an interaction between age, BMI, and recent knee injury, and if we could identify a subset of individuals who were more likely to develop AKOA instead of 
common KOA. While we pay a lot of attention to injuries in young physically active patients, findings in this study reminds us that we must also carefully monitor older and overweight patients who report a recent knee injury. Unfortunately, we were limited to using self-reported injury data. It will be important for future studies to clarify what injuries are occurring in these different subsamples and to determine which injuries increase the risk of AKOA.

While older age and greater BMI are independently associated with accelerated KOA, we found that older individuals who had a higher BMI were more likely to develop AKOA than common KOA. We should be especially concerned when older individuals report a history of a recent knee injury. These patients may need to be closely monitored for at least the next 12 months to monitor for the onset of accelerated KOA. Hopefully, future studies will enable us to better understand why these individuals are at risk for accelerated KOA so that we can optimize our therapeutic approach.

\section{Acknowledgments}

These analyses were funded by the National Institutes of Health (HHSN268201200032C, Eaton \& R01AR065977-01A1, Driban). The Osteoarthritis Initiative is a public-private partnership comprised of five contracts (N01-AR-2-2258; N01-AR-2-2259; N01-AR-2-2260; N01-AR-2-2261; N01-AR-2-2262) funded by the National Institutes of Health, a branch of the Department of Health and Human Services, and conducted by the OAI Study Investigators. Private funding partners include Merck Research Laboratories; Novartis Pharmaceuticals Corporation, GlaxoSmithKline; and Pfizer, Inc. Private sector funding for the OAI is managed by the Foundation for the National Institutes of Health. This manuscript was prepared using an OAI public use data set and does not necessarily reflect the opinions or views of the OAI investigators, the $\mathrm{NIH}$, or the private funding partners.

This work was also supported in part by the Houston Veterans Affairs Health Services Research and Development Center of Excellence (HFP90-020). The views expressed in this article are those of the author(s) and do not necessarily represent the views of the Department of Veterans Affairs.

\section{References}

1. Driban JB, Eaton CB, Lo GH, Ward RJ, Lu B, McAlindon TE. Association of knee injuries with accelerated knee osteoarthritis progression: data from the Osteoarthritis Initiative. Arthritis Care Res. 2014; 66:1673-1679.10.1002/acr.22359

2. Driban JB, Price LL, Eaton CB, Lu B, Lo GH, Lapane KL, McAlindon TE. 2015Individuals with incident accelerated knee osteoarthritis have greater pain than those with common knee osteoarthritis progression: data from the Osteoarthritis Initiative. Clin Rheumatol. 10.1007/ s10067-015-3128-2

3. Peterfy CG, Schneider E, Nevitt M. The osteoarthritis initiative: report on the design rationale for the magnetic resonance imaging protocol for the knee. Osteoarthritis Cartilage. 2008; 16:14331441.10.1016/j.joca.2008.06.016 [PubMed: 18786841]

4. [Accessed 08 December 2015] The Osteoarthritis Initiative. http://oai.epi-ucsf.org/

5. Ferrandiz ML, Terencio MC, Ruhi R, Verges J, Montell E, Torrent A, Alcaraz MJ. Influence of age on osteoarthritis progression after anterior cruciate ligament transection in rats. Exp Gerontol. 2014; 55:44-48.10.1016/j.exger.2014.03.010 [PubMed: 24667123]

6. Wu CL, Jain D, McNeill JN, Little D, Anderson JA, Huebner JL, Kraus VB, Rodriguiz RM, Wetsel WC, Guilak F. Dietary fatty acid content regulates wound repair and the pathogenesis of osteoarthritis following joint injury. Ann Rheum Dis. 201410.1136/annrheumdis-2014-205601

7. Mooney RA, Sampson ER, Lerea J, Rosier RN, Zuscik MJ. High-fat diet accelerates progression of osteoarthritis after meniscal/ligamentous injury. Arthritis Res Ther. 2011; 13:R198.10.1186/ar3529 [PubMed: 22152451] 
8. Driban JB, Barr AE, Amin M, Sitler MR, Barbe MF. Joint inflammation and early degeneration induced by high force reaching are attenuated by ibuprofen in an animal model of work-related musculoskeletal disorder. J Biomed Biotechnol. 2011; 2011:691412. [PubMed: 21403884]

9. Kerkhof HJ, Bierma-Zeinstra SM, Castano-Betancourt MC, de Maat MP, Hofman A, Pols HA, Rivadeneira F, Witteman JC, Uitterlinden AG, van Meurs JB. Serum C reactive protein levels and genetic variation in the CRP gene are not associated with the prevalence, incidence or progression of osteoarthritis independent of body mass index. Ann Rheum Dis. 2010; 69:1976-1982.10.1136/ ard.2009.125260 [PubMed: 20511616]

10. Lucey AJ, Paschos GK, Thorsdottir I, Martinez JA, Cashman KD, Kiely M. Young overweight and obese women with lower circulating osteocalcin concentrations exhibit higher insulin resistance and concentrations of C-reactive protein. Nutr Res. 2013; 33:67-75.10.1016/j.nutres.2012.11.011 [PubMed: 23351412]

11. Labouesse MA, Gertz ER, Piccolo BD, Souza EC, Schuster GU, Witbracht MG, Woodhouse LR, Adams SH, Keim NL, Van Loan MD. Associations among endocrine, inflammatory, and bone markers, body composition and weight loss induced bone loss. Bone. 2014; 64:138-146.10.1016/ j.bone.2014.03.047 [PubMed: 24709689]

12. Jonsson H, Helgadottir GP, Aspelund T, Eiriksdottir G, Sigurdsson S, Siggeirsdottir K, Ingvarsson T, Harris TB, Launer L, Gudnason V. Hand Osteoarthritis Severity is Associated with Total Knee Joint Replacements Independently of BMI. The Ages-Reykjavik Study. Open Rheumatol J. 2011; 5:7-12.10.2174/1874312901105010007 [PubMed: 21552415]

13. Kraus VB, Stabler TV, Luta G, Renner JB, Dragomir AD, Jordan JM. Interpretation of serum Creactive protein (CRP) levels for cardiovascular disease risk is complicated by race, pulmonary disease, body mass index, gender, and osteoarthritis. Osteoarthritis Cartilage. 2007; 15:966971.10.1016/j.joca.2007.02.014 [PubMed: 17395501]

14. Chen HC, Shah S, Stabler TV, Li YJ, Kraus VB. Biomarkers associated with clinical phenotypes of hand osteoarthritis in a large multigenerational family: the CARRIAGE family study. Osteoarthritis Cartilage. 2008; 16:1054-1059.10.1016/j.joca.2007.12.010 [PubMed: 18291686]

15. Lee SW, Jo HH, Kim MR, You YO, Kim JH. Association between obesity, metabolic risks and serum osteocalcin level in postmenopausal women. Gynecol Endocrinol. 2012; 28:472_ 477.10.3109/09513590.2011.633660 [PubMed: 22114890]

16. Rosa SC, Rufino AT, Judas FM, Tenreiro CM, Lopes MC, Mendes AF. Role of glucose as a modulator of anabolic and catabolic gene expression in normal and osteoarthritic human chondrocytes. J Cell Biochem. 2011; 112:2813-2824.10.1002/jcb.23196 [PubMed: 21608018] 
No Knee Osteoarthritis

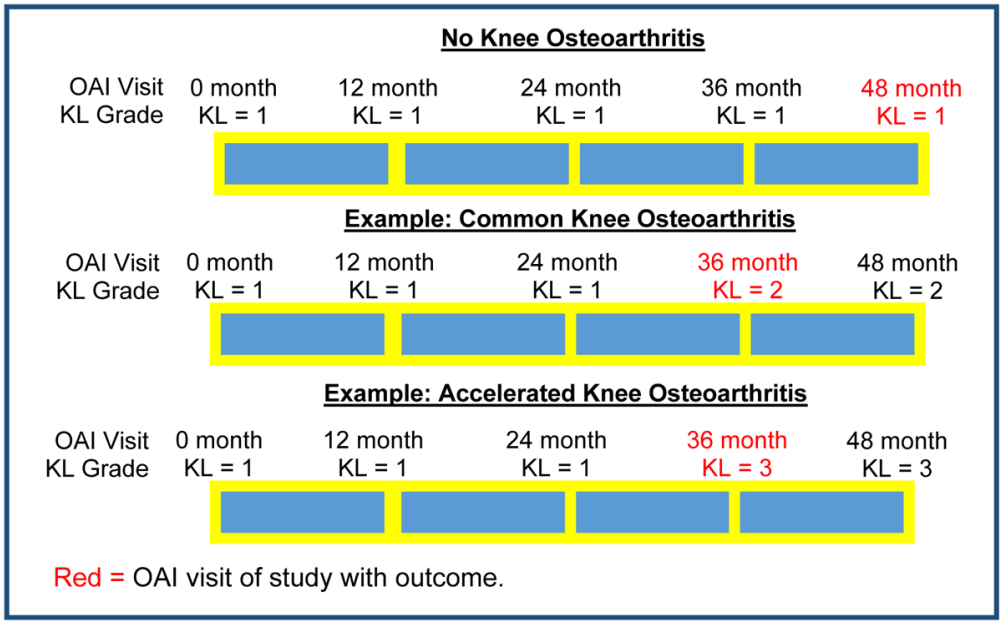

Fig. 1.

Examples of Study Outcome Visits 
A

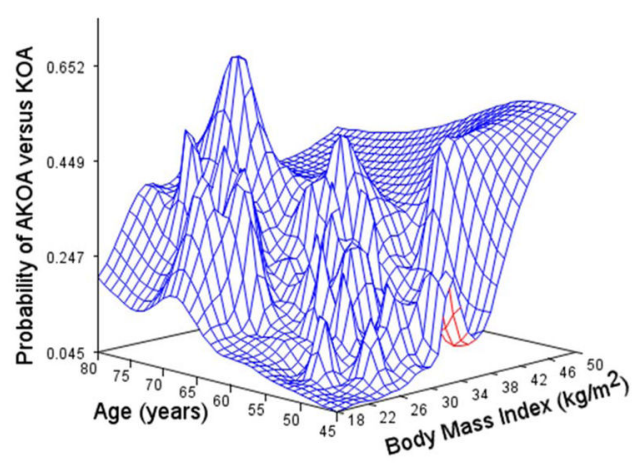

B

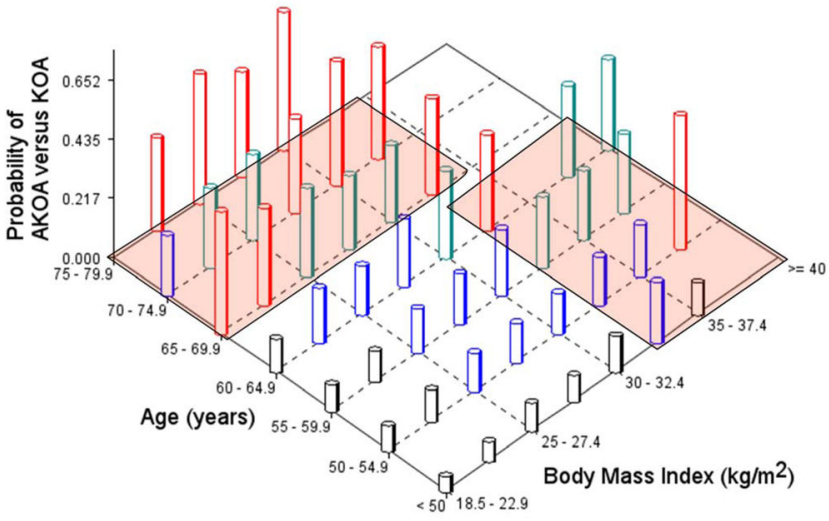

Fig. 2. Probability of Accelerated Knee Osteoarthritis (AKOA) Compared with Common Knee Osteoarthritis (KOA) by Age and Body Mass Index

(a) Surface plot of the probability of AKOA (vertical axis) by age and body mass index as continuous variables. (b) Surface plot of the probability of AKOA (vertical axis) by age and body mass index categorized by World Health Organization criteria. Red boxes highlight high-risk subsets of interest ( $>65$ years with BMI $<35 \mathrm{~kg} / \mathrm{m} 2,<65$ years with BMI $>32.5$ $\mathrm{kg} / \mathrm{m} 2$ ) 


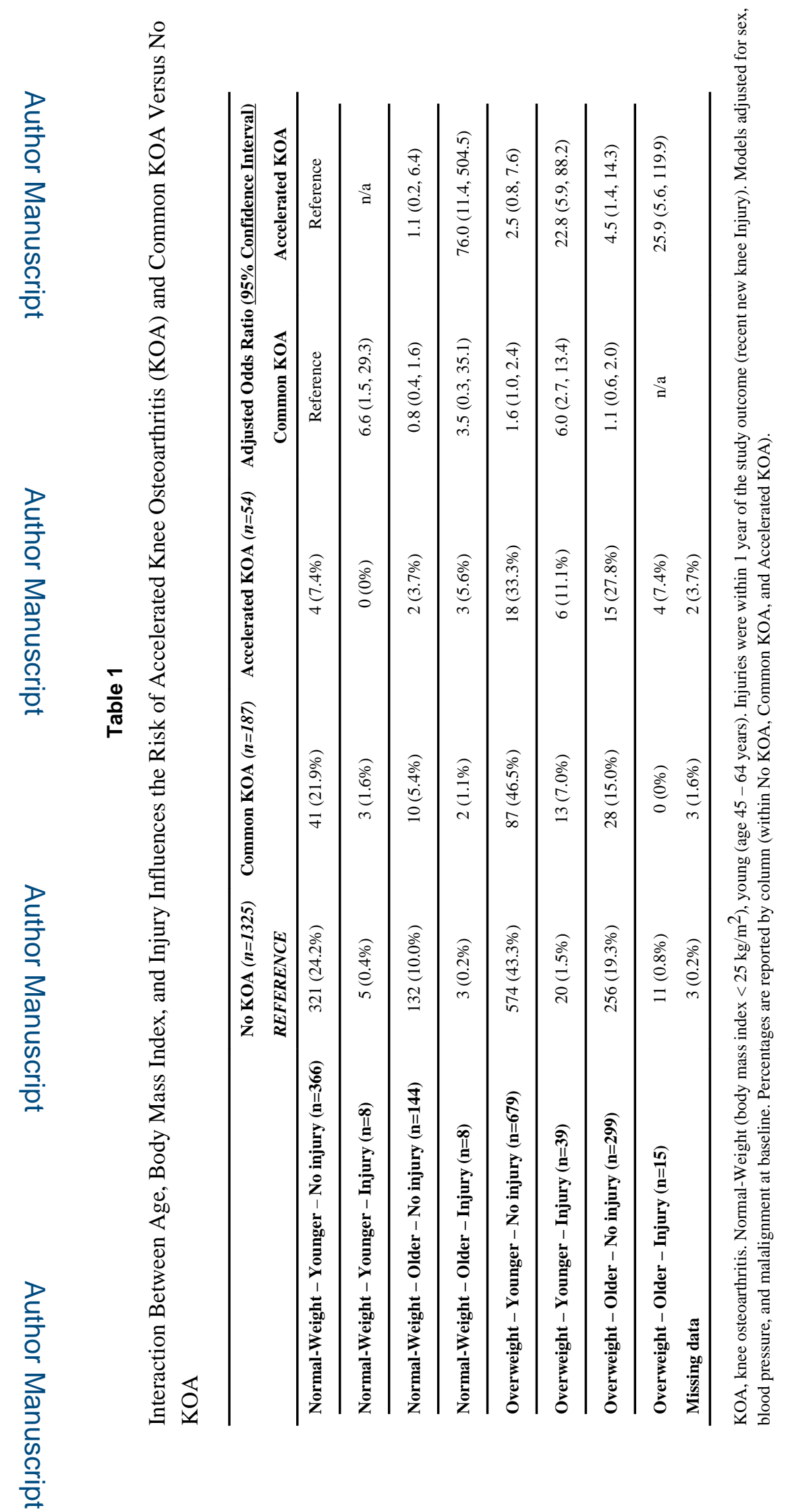

Clin Rheumatol. Author manuscript; available in PMC 2017 April 01. 


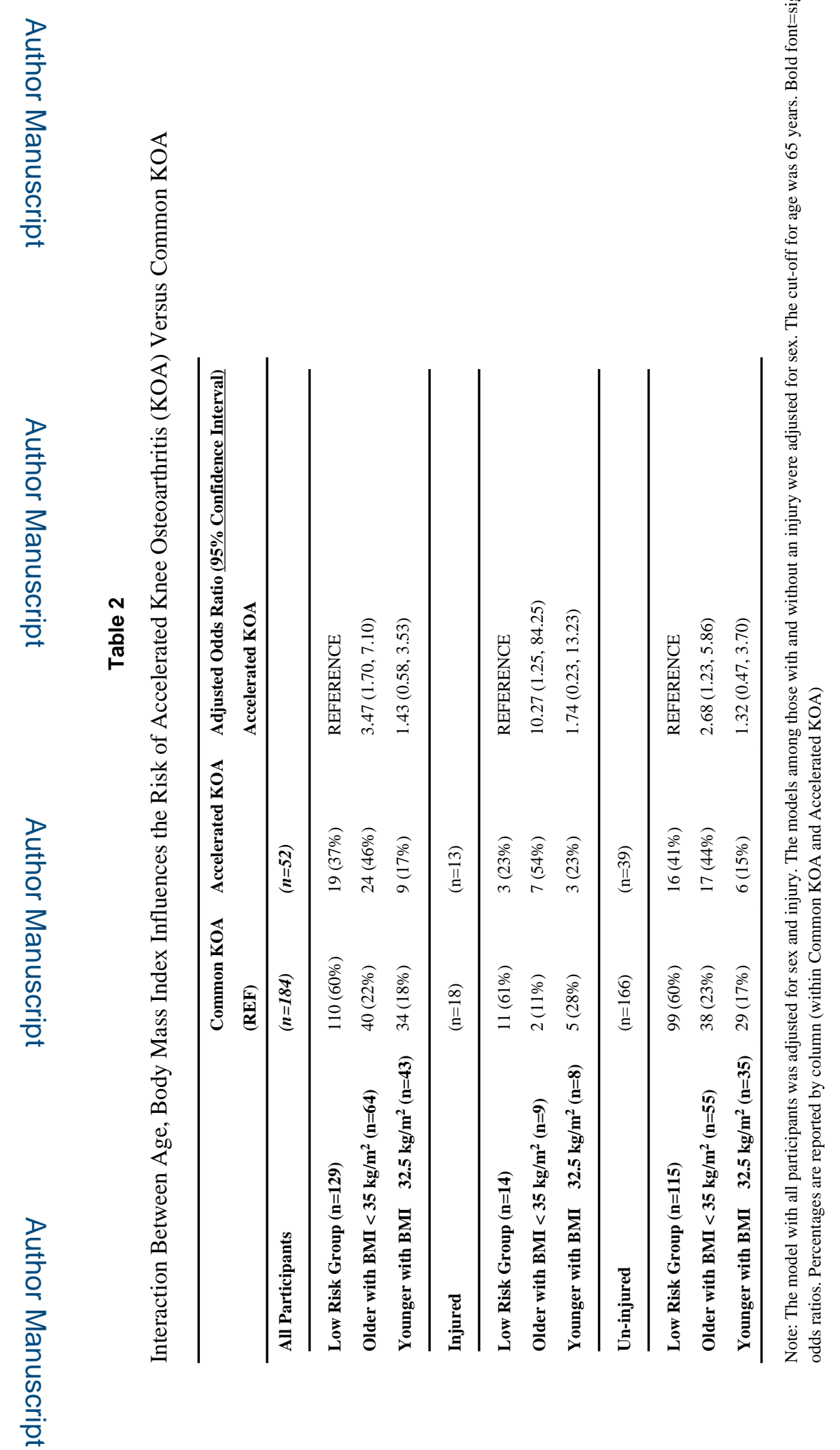

Clin Rheumatol. Author manuscript; available in PMC 2017 April 01. 